\title{
The discovery of a novel snake neurotoxin, candoxin - implications for cholinergic signalling mechanisms in health and disease
}

\author{
Selvanayagam Nirthanan ${ }^{1}$ \\ Journal of the Ceylon College of Physicians, 2013, 44, 14-21
}

\section{Introduction}

\section{Animal toxins: key players in science and medicine}

Venomous animals, in the course of their long evolutionary history, have created innovative and intricate protein structural motifs to engender a vast resource of pharmacologically novel peptide toxins that target a wide variety of receptors and ion channels with high affinity and exquisite specificity ${ }^{1,2}$. While early venom research was motivated by our desire for effective cures for snake envenomation, our perspectives on animal toxins have changed dramatically due to accumulating data that has revealed a far wider scope for these natural biomolecules, which have assumed great significance as molecular probes and pharmacological tools to investigate the functional biology of receptors and ion channels as well as providing lead compounds for the design of clinically useful drugs ${ }^{1,3}$. There is perhaps no better example to highlight the significant contributions made by venom peptides to science and medicine than the discovery, about fifty years ago, of the curaremimetic neurotoxin, $\alpha$-bungarotoxin from the venom of the Taiwanese banded krait (Bungarus multicinctus) ${ }^{4}$, which enabled the localization of the nicotinic acetylcholine receptor, ultimately making it one of the most thoroughly characterized receptors today, and in the process enhancing our knowledge of the pathphysiology of myasthenia gravis ${ }^{4,6}$.

\section{Snake envenomation: nefarious role of neurotoxins}

Snake venoms are a cocktail of hundreds of toxins and enzymes that have optimally evolved as a lethal weapon for predation as well as defense against predators. Depending on the species, snake envenomation in humans may result in peripheral neuro-

\footnotetext{
${ }^{1}$ School of Medicine and School of Medical Science, Griffith University, Queensland, Australia. Molecular Basis of Disease Program, Griffith Health Institute, Queensland, Australia. Yong Loo Lin School of Medicine, National University of Singapore, Singapore.
}

toxicity, coagulative disorders, myotoxicity, renal failure as well as severe necrosis at the site of the bite, all of which can be potentially fatal ${ }^{7,8}$. Snake envenomation is a major clinical problem with an estimated 5.5 million cases of snake bites reported worldwide in parts of Asia, Africa and Latin America, where the annual mortality is estimated to be up to $125,000^{9,10}$. Sri Lanka in particular has one of the highest incidence rates of venomous snake bites ${ }^{11}$. A prime target of snake toxins is the mammalian nervous system, particularly the skeletal muscle neuromuscular junction, where neurotransmission is inhibited leading to paralysis of skeletal muscles including those of respiration ${ }^{7,12}$. Clearly therefore, the understanding of this fundamental pathology at a molecular level is of great clinical significance.

\section{Snake neurotoxins which target cholinergic neurotransmission}

The arsenal of snake neurotoxins that interfere with cholinergic neurotransmission may selectively target a multitude of subtypes of nicotinic and muscarinic acetylcholine receptors at peripheral, central and extra-neuronal sites ${ }^{2}$. Often, a combination of many types of neurotoxins may be present together in the venom of one species. The principal neurotoxic components of Elapid (cobras, kraits, mambas, coral snakes and Australian elapids) and Hydrophiid (sea snakes) snake venoms are curaremimetic or $\alpha$ neurotoxins that disrupt neuromuscular transmission by inhibition of postsynaptic nicotinic acetylcholine receptors $^{2}$. The skeletal muscle neuromuscular junction is also susceptible to presynaptic neurotoxins (e.g. $\beta$-bungarotoxin) which contain phospholipase $A_{2}$ enzymes as an integral part of the neurotoxin complex and, essentially, mediate their neurotoxicity by inhibiting the release of acetylcholine. Other neurotoxins that interfere with cholinergic neurotrans-mission include fasciculins from mamba (Dendroaspis spp.) venoms that inhibit the activity of acetylcho-linesterase present at the neuromuscular junction and central synapses and $\kappa$-neurotoxins (Bungarus spp.) which primarily bind to neuronal $\alpha 3 \beta 2$ nicotinic receptors. Several muscarinic toxins have also been isolated from mamba venoms (Dendroaspis spp.), which are 
antagonists or agonists at various subsets of muscarinic receptors in the brain as well as at peripheral sites (reviewed in ${ }^{13}$ )

\section{Curaremimetic $\alpha$-neurotoxins: more than just a case of mimicry}

Curaremimetic or $\alpha$-neurotoxins mimic the neuromuscular blocking effects of the plant alkaloid (+)-tubocurarine, albeit with greater affinity and poor reversibility of action. Hence, they are referred to as curaremimetic neurotoxins or postsynaptic neurotoxins to reflect their post-junctional site of action at the neuromuscular junction or simply as $\alpha$-neurotoxins, a suffix of historical significance ${ }^{14}$. It must be emphasised that snake venoms are not the exclusive source of $\alpha$ neurotoxins. The venoms of marine cone snails also represent a rich combinatorial-like library of evolutionarily selected, pharmacologically active conotoxins that target a wide variety of receptors and ion-channels, including a number of nicotinic receptor subtypes (reviewed in ${ }^{15,16}$ ).

\section{The three-finger toxin scaffold: three fingers in many pies}

Snake $\alpha$-neurotoxins belong to the three-finger toxin superfamily of non-enzymatic polypeptides containing 60-74 amino acid residues. They have a distinctive protein structure formed by three adjacent loops (like three outstretched fingers) that emerge from a globular core which is cross-linked by four conserved disulfide bridges ${ }^{2,13}$. The three-finger fold is amenable to a variety of overt and subtle deviations, such as the size of the loops and C-terminal tail as well as twists and turns of various loops, all of which may have great significance with respect to functional diversity and selectivity of molecular targets. Hence, despite the similar overall fold, three-finger toxins demonstrate an assorted range of pharmacological activities including, but not limited to, peripheral and central neurotoxicity, cyotoxicity, cardiotoxicity, inhibition of enzymes such as acetylcholinesterase and proteinases, hypotensive effect and platelet aggregation ${ }^{2,13}$. It thus appears that snakes adhere to a policy of structural economy by utilizing a limited number of molecular molds to achieve remarkable functional diversity.

\section{Snake $\alpha$-neurotoxins: the long and short of it}

Despite their common structural fold, $\alpha$-neurotoxins are classified as short-chain neurotoxins (e.g. erabutoxin-b) that have 60-62 residues and 4 conserved disulfide bonds and long-chain neurotoxins (e.g. $\alpha$ bungarotoxin; $\alpha$-cobratoxin) with $66-75$ residues and 5 disulfide bonds ${ }^{2}$. The additional disulfide bridge in long-chain $\alpha$-neurotoxins, as well in the neuronal $\alpha$ bungarotoxin is located in loop 2 . This fifth bridge, which cyclizes a helix-like conformation at the tip of loop 2, has been reported to be crucial for long chain $\alpha$ - and $\kappa$-neurotoxins to bind to $\alpha 7$ and $\alpha 3 \beta 2$ neuronal, but not to muscle $(\alpha \beta \gamma \delta)$, nicotinic receptors ${ }^{17}$. The nonconventional neurotoxins constitute a new class of three-finger neurotoxins that are structurally and functionally distinct from typical long and short $\alpha$ neurotoxins ${ }^{18}$. Candoxin, the best characterised member of this family is the focus of this Oration Paper.

\section{Methodology}

The isolation and purification of peptide toxins from animal venoms is a well-established, widely utilized and streamlined protocol in our laboratory ${ }^{19-23}$. Lyophilised Malayan krait (Bungarus candidus) venom was subjected to multi-stage high performance liquid chromatography (HPLC) to isolate and purify a novel neurotoxin that was subsequently characterised as candoxin. The primary structure of candoxin was determined by electrospray ionization mass-spectrometry and matrix-assisted laser desorption ionization - time of flight (MALDI-TOF) mass-spectrometry and its primary $\mathrm{N}$-terminal amino acid sequence was determined by automated Edman degradation ${ }^{19}$. The tertiary structure of candoxin was determined by two established approaches using $\mathrm{x}$-ray crystallography as well as nuclear magnetic resonance $(\mathrm{NMR})^{24,25}$. The pharmacological characterisation of candoxin was carried out in vitro, on isolated tissues in organ bath studies using nerve-muscle preparations that are well-established representative models of the mammalian neuromuscular junction ${ }^{2,26}$ as well as in vivo, in anaesthetised rodents ${ }^{20}$. To identify candoxin's molecular target(s) and understand its interactions at receptor / ion-channel level, electrophysiological studies were conducted utilising the two-electrode voltage clamp with Xenopus oocytes as the expression system for expressing the variety of potential target receptors ${ }^{19,20,27,28}$

\section{Results}

\section{Isolation and purification of candoxin}

Candoxin was purified to homogeneity by multistage HPLC, the name candoxin denoting the peptide as a toxin derived from Bungarus candidus venom ${ }^{19}$. To ensure the absence of contaminants, especially by other neurotoxin(s) present in the venom, the purified sample of candoxin was subjected to several sensitive assays and found to be homogenous by analytical reverse-phase HPLC, capillary electrophoresis as well as by ESI- and MALDI-TOF mass spectrometry. Candoxin has a molecular mass of $7334.67 \pm 0.35$ and constitutes about $1-2 \%$ of the crude venom. We were able to unequivocally identify all the residues and determine the complete amino acid sequence of candoxin (SWISS-PROT protein database accession number P81783), which was found to be a polypeptide 
consisting of 66 amino acid residues including 10 cysteine residues that corresponded to five disulfide linkages. The disulfide linkages, established by studies of its tertiary structure ${ }^{24,25}$, showed the presence of five disulfide bridges, four of which were homologous to the four conserved disulfide bridges found in other members of the three-finger toxin family. The fifth disulfide bridge in candoxin is uniquely located at the tip of loop I (Cys6-Cys11) instead of in loop II as found in other snake $\alpha$-neurotoxins. Candoxin shares just $-30-40 \%$ homology with other known snake $\alpha$ neurotoxins, as well as other non-neurotoxic snake three-finger toxins. Much of this homology can be attributed to the four conserved disulfide bridges suggesting that any similarity extends just to the overall protein fold and not to the critical amino acid residues that determine pharmacological activity.

\section{Neuromuscular toxicity produced by candoxin in vitro and in vivo}

Mice injected intraperitoneally with candoxin showed flaccid paralysis of the hind limbs and death attributable to respiratory paralysis mimicking symptoms of human envenomation by Elapid snakes. Accordingly, we screened candoxin for biological activity on isolated nerve-muscle preparations, where it produced a rapid, concentration-dependent neuromuscular blockade which was postsynaptic in nature. There was no pharmacological or histological evidence of myotoxicity. The neuromuscular blockade was sustained for over $90 \mathrm{~min}$ without spontaneous reversal following which the contractile responses of the muscle evoked by electrical stimulation were rapidly and completely restored by washing out the toxin from the organ bath chamber with fresh Kreb's physiological saline. In another series of experiments, neostigmine, an acetylcholine esterase inhibitor, produced complete reversal of the neuromuscular blockade produced by candoxin. In contrast, other known snake neurotoxins such as erabutoxin-b, $\alpha$-bungarotoxin and $\alpha$-cobratoxin produced neuromuscular blockade that was $\sim 5$ fold more potent than that produced by candoxin $\left(\mathrm{IC}_{50} \sim 1.5\right.$ $\mu \mathrm{M})$ but which was virtually irreversible in their action.

The pharmacological actions of candoxin in anaesthetised rats mirrored its effects seen in vitro ${ }^{20}$. Candoxin produced dose-dependent, complete blockade of nerve-evoked twitch responses of the tibialis anterior muscle of the leg, with complete blockade occurring at a dose of $1 \mathrm{mg} / \mathrm{kg}$ of candoxin. The twitch responses of the muscle recovered spontaneously and completely with the time taken for complete spontaneous recovery increasing proportionately with increasing doses of candoxin. The neuromuscular blockade produced by candoxin was also completely reversed by the injection of neostigmine $(0.4 \mu \mathrm{g} / \mathrm{kg})$. Candoxin did not appear to affect the arterial blood pressure, heart rate and cardiac conductivity of the anaesthetized rat during neuromuscular blockade. In contrast to candoxin, erabutoxin-b and $\alpha$-bungarotoxin produced rapid neuromuscular blockade in the tibialis anterior muscle that did not recover spontaneously nor was the blockade reversed by neostigmine ${ }^{20}$.

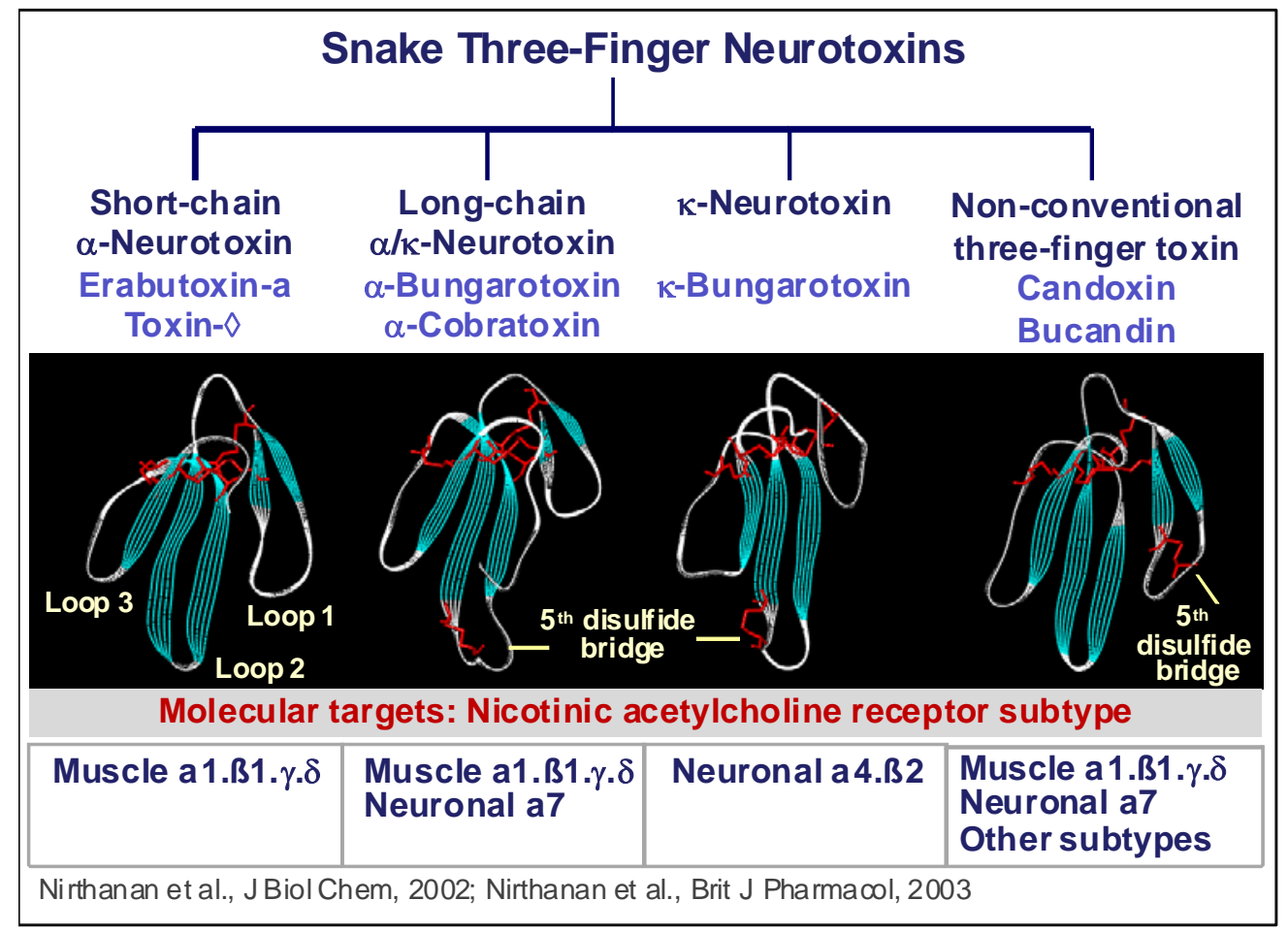

Figure 1. 


\section{Electrophysiological studies on nicotinic acetylcholine receptors}

Electrophysiological experiments on various subtypes of nicotinic acetylcholine receptors expressed in Xenopus oocytes were designed to elucidate the molecular target(s) of candoxin. Candoxin strongly inhibited acetylcholine evoked currents in the muscle $(\alpha \beta \gamma \delta)$ nicotinic receptors $\left(\mathrm{IC}_{50}=\sim 10 \mathrm{nM}\right)$ with comparable affinity as $\alpha$-bungarotoxin $\left(\mathrm{IC}_{50}=\sim 5 \mathrm{nM}\right)^{19}$. In congruence with organ bath studies, recovery from candoxin-induced receptor blockade was rapid and complete following a 10 minute wash; whereas, $\alpha$-bungarotoxin-induced inhibition of the receptor was irreversible. Additionally, candoxin also inhibited acetylcholine evoked currents in oocytes expressing the $\alpha 7$ neuronal subtype of nicotinic receptors, the ability to target $\alpha 7$ receptors resembling the pharmacological profile of other snake long neurotoxins such as $\beta$-bungarotoxin and $\alpha$-cobratoxin ${ }^{19}$.

\section{Three-dimensional structural studies on candoxin}

The three-finger snake toxin family is characterized by a distinctive protein scaffold formed by three adjacent loops that emerge from core region which is crosslinked by four conserved disulfide bridges (for reviews, see $^{2,13,18}$ ). The 3D tertiary structure of candoxin (PDB \# 1JGK) revealed that the fifth disulfide bridge in candoxin is located at the tip of loop I instead of in loop II as found in other long-chain $\alpha$-neurotoxins ${ }^{19,} 24,29,30$ (see inset figure 1.) ${ }^{24,25}$. This structural motif of candoxin with its unique disulfide bridge pairing, places it within a new family of non-conventional toxins ${ }^{18}$.

\section{Discussion}

\section{Candoxin, a novel toxin belonging to a new family of snake neurotoxins}

The three-finger protein scaffold is utilised by snakes as a molecular mould or template to generate an arsenal of toxins (including neurotoxins) ${ }^{27-30}$, that have distinct pharmacological properties enabled by subtle modifications in the template's polypeptide chain length, twists and turns of the three loops as well as amino acid substitutions in the primary sequence ${ }^{2,18}$. The unique structural motif of candoxin with its uniquely placed fifth disulfide bridge at the tip of loop I instead of in loop II19,24,29,30, warranted its place in a new class of snake neurotoxins which were "non-conventional" in structure ${ }^{18}$. At the time of discovery of candoxin, another toxin (bucandin; PDB \# 1IJC) was also isolated from the same venom source in our laboratory ${ }^{31}$. While the pharmacology and molecular target of bucandin remains undetermined at this time, structurally, it also belongs to the new family of non-conventional toxins as candoxin ${ }^{18,31}$.
At present, about 30 amino acid sequences of non-conventional toxins have been identified either from their cDNA sequences or by their isolation from venoms. More recently, we have also described and characterised, a novel "conjoint twin" of a nonconventional neurotoxin - named irditoxin from the venom of the brown tree-snake Boiga irregularis ${ }^{29}$. This is the first example of a snake neurotoxin that is a dimeric molecule where the two subunits are covalently linked by an inter-chain disulfide bond. Interestingly, irditoxin showed taxon-specific for birds and lizards and was ineffective at the mammalian neuromuscular junction, suggesting that this toxin may have evolved to optimally target the natural prey of this arboreal snake species ${ }^{29}$. The ability of non-conventional neurotoxins to target different subtypes of nicotinic acetylcholine receptors and distinguish between receptors of different species makes them a unique resource for the development of molecular probes and potential drug leads.

\section{Pharmacological implications of candoxin's novel structure}

Notwithstanding their classification as short- and long-chain neurotoxins, both types of curaremimetic snake $\alpha$-neurotoxins bind with high affinity to the muscle $(\alpha \beta \gamma \delta)$ nicotinic acetylcholine receptor, which underpins much of the clinical neurotoxicity observed in human envenomation by Elapids. In contrast, only long-chain neurotoxins are able to recognize the neuronal $\alpha 7$ nicotinic receptor with high affinity. This pharmacological distinction has been attributed to the presence of a small helix-like segment cyclized by the fifth disulfide bridge located at the tip of the middle loop of long-chain neurotoxins that is lacking in shortchain neurotoxins ${ }^{17,32,33}$. Clearly therefore, the two families of curaremimetic toxins, which share many structural similarities, are not functionally homogenous.

Interestingly, candoxin which also blocks both, the muscle $(\alpha \beta \gamma \delta)$ and $\alpha 7$ receptors at low nanomolar concentrations, showed distinct differences from longchain neurotoxins with respect to structure and function. Structurally, the first loop of candoxin was found to be longer than that of typical long-chain neurotoxins while it also lacked the long carboxyterminal tail that is a characteristic feature of most long-chain neurotoxins. Functionally, although candoxin lacks the helix-like conformation of the tip of the middle loop seen in long-chain neurotoxins and hitherto considered essential for high-affinity binding to $\alpha 7$ receptors $^{17}$, it blocks $\alpha 7$ receptors at comparable low nanomolar concentrations. We have also identified the putative amino acid residues in candoxin which likely contribute to the recognition of the muscle and neuronal $\alpha 7$ nicotinic receptors ${ }^{18,19}$. 


\section{Novel reversible neuromuscular blockade produced by candoxin}

Candoxin produced rapid onset, dose- and timedependent neuromuscular blockade in mammalian skeletal muscle attributable to competitive inhibition of postsynaptic nicotinic acetylcholine receptors at the neuromuscular junction ${ }^{19,20}$. These effects closely resemble the neuromuscular blockade produced by curaremimetic $\alpha$-neurotoxins such as erabutoxin- $b, \alpha-$ bungarotoxin and $\alpha$-cobratoxin, which are well documented to have high selectivity and high affinity for postsynaptic nAChRs . A direct myotoxic effect of candoxin was excluded pharmacologically ${ }^{19}$, biochemically ${ }^{20}$ and histologically since myotoxic snake venoms could also cause a failure of muscle contraction due to a direct destructive action of enzymes such as phospholipase $\mathrm{A}_{2}{ }^{34}$.

In contrast to most snake $\alpha$-neurotoxins that produce virtually irreversible neuromuscular blockade ${ }^{35}$, candoxin produced a blockade of nerve-evoked twitch responses in vitro and in vivo that was rapidly and completely reversed by washing or by the addition of the anticholinesterase neostigmine ${ }^{19,20}$. Hence, the neuromuscular blockade produced by candoxin closely resembled the neuromuscular effects of d-tubocurarine, a reversible and competitive antagonist of postsynaptic nicotinic acetylcholine receptors and the precursor to neuromuscular blockers in current clinical use. Some cone snail neurotoxins ( $\alpha$-conotoxins $\mathrm{MI}$ and $\mathrm{Gl}$ ), short ( $12-30$ residues) disulfide-rich peptides, are well known to produce reversible postsynaptic neuromuscular blockade in vitro and in vivo ${ }^{36}$. There are also a few other neurotoxins and neurotoxin-homologues isolated from snake venoms which reportedly exhibited reversible or partially reversible neuromuscular blockade in vitro. Candoxin showed very little amino acid sequence homology to all these 'reversible' toxins and to the best of our knowledge, it is one of two of the most rapidly reversible curaremimetic snake neurotoxins.

It could be argued that the reversibility of neuromuscular blockade induced by some toxins, as opposed to the irreversible blockade attributed to others, may just result from their weak binding affinity to the receptors. However, in electrophysiological studies, $\alpha$-bungarotoxin produced an irreversible block of muscle $(\alpha \beta \gamma \delta)$ receptors with comparable affinity as candoxin $\mathrm{IC}_{50}(\sim 5-10 \mathrm{nM})$, which produced reversible blockade of the same receptor ${ }^{19}$. Clearly, therefore, the reversibility of toxin action at the neuromuscular junction is not always a reflection of their binding affinity to the receptor. It has been suggested that reversibility of neurotoxin action may be associated with a specific area of interaction on the toxin molecule, distinct from the receptor recognition site and it is postulated that the absence of a conserved aspartate at position 31 in the neurotoxin amino acid sequence may be associated with easy reversibility of neuromuscular blockade produced by snake neurotoxins. Interestingly, in support of this hypothesis, candoxin and fulditoxin also lack an aspartate at a position homologous to position 31 in their amino acid sequences ${ }^{20}$.

\section{Train-of-four fade induced by candoxin during neuromuscular blockade}

Candoxin, like that observed with tubocurarinelike neuromuscular blockers in clinical settings ${ }^{37}$, produced significant "train-of-four fade" during the onset of and recovery from neuromuscular blockade in vitro and in anaesthetized rats. The "fade" phenomenon is characterized by rapid rundown in the successive twitch responses to train-of-four nerve stimulation ${ }^{20}$. In contrast, most typical snake neurotoxins like erabutoxin- $b, \alpha$-cobratoxin and $\alpha$-bungarotoxin did not produce such a fade during neuromuscular blockade ${ }^{37}$. The phenomenon of train-of-four fade has been ascribed to inhibition of presynaptic nAChRs at the neuromuscular junction which sustain an autofacilitatory positive feedback mechanism ${ }^{37}$. Hence, in addition to its established competitive antagonistic action at postsynaptic nicotinic acetylcholine receptors, candoxin also mediates a presynaptic action at the neuromuscular junction. This is a novel and unusual functional characteristic of candoxin, not previously reported for snake toxins that exert a curaremimetic effect at the neuromuscular junction.

\section{A twist in the tale: mammalian homologues of snake neurotoxins}

Interestingly, proteins outside of the well-established snake venom superfamily, also adopt the threefinger fold. These constitute members of the $L y-6$ superfamily and include mammalian immune cell surface proteins, human complement regulatory protein CD59, urokinase-type plasminogen activator receptor and prostate stem cell antigen; all of which are tethered membrane proteins ${ }^{38}$. While most $L y-6$ superfamily proteins bore no similarity in function to snake neurotoxins, mammalian endogenous proteins Lynx1 and Lynx2, were found to be novel modulators of neuronal $\alpha 4 \beta 2$ and $\alpha 7$ nAChRs by enhancing agonist sensitivity and altering desensitization kinetics in vitro. Lynx1and Lynx2 have also been found to play critical roles in cholinergic signaling in vivo, in the brain and outside the nervous system, exhibiting short-term enhancement of learning ${ }^{39}$, implicated in anxiety behaviours ${ }^{40}$ and cholinergic signaling in the airway epithelium in response to nicotine ${ }^{41}$. SLURP-1 and SLURP-2 first identified in human keratinocytes, were the first examples of endogenously secreted three-finger proteins (unlike the tethered Lynx proteins), and acted as positive allosteric modulators of $\alpha 7$ nicotinic 


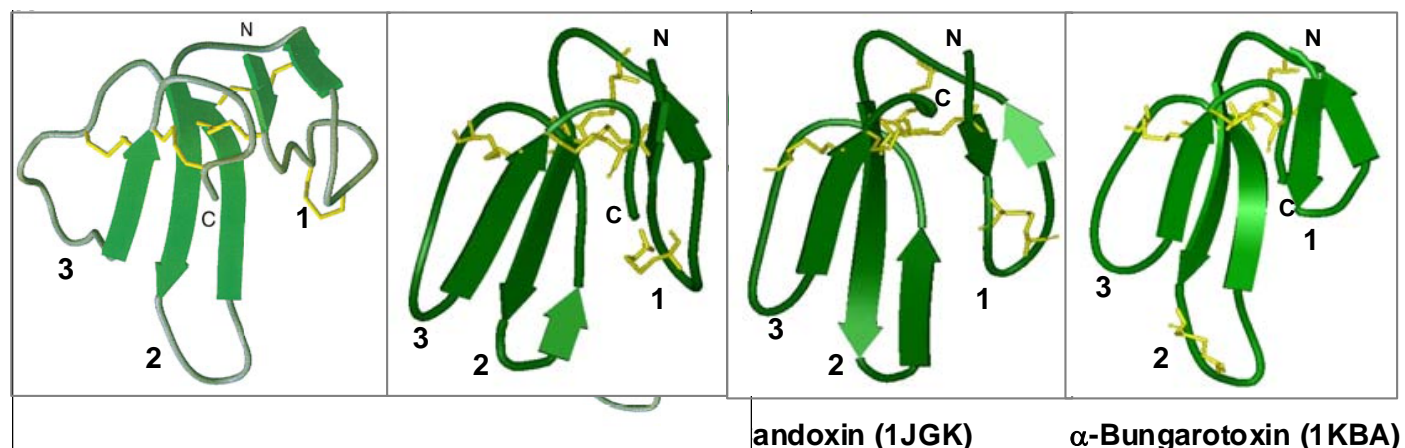

Molecular model of murine Lynx1 (65) in comparison with experimental structures of nonconventional neurotoxins, bucandin and candoxin (31) and the typical $\alpha$-neurotoxin $\alpha$-bungarotoxin. The $5^{\text {th }}$ disulfide bridge is located in the N-terminal loop-1 of Lynx1 and non-conventional neurotoxins, whereas it is in loop- 2 in typical $\alpha$-neurotoxins.

Figure 2.

receptors and these have been implicated in the wound healing mechanism ${ }^{42}$.

This evidence points to common molecular targets in the cholinergic circuitry for mammalian Lynx and SLURP proteins and their snake neurotoxin cousins. Hence, the Lynx and SLURP proteins are considered to be "prototoxins" - natural, structural, functional and evolutionarily-related mammalian homologues to snake neurotoxins ${ }^{43}$. Interestingly, among the classes of snake neurotoxins, the non-conventional neurotoxins ${ }^{18}$ such as candoxin ${ }^{19}$ and bucandin ${ }^{31}$ are closest in structure to these endogenous prototoxins (see inset figure 2), having the identical disulfide bridge motif (with the $5^{\text {th }}$ disulfide bridge in the first loop).

\section{Conclusion}

In conclusion, candoxin is structurally distinct from typical snake $\alpha$-neurotoxins and hence belongs to a new class of non-conventional three-finger neurotoxins. The most salient structural distinction, with functional implications, is the presence of a fifth disulfide bridge in the first ( $\mathrm{N}$-terminal) loop in non-conventional neurotoxins instead of the second (middle) loop as in typical snake $\alpha$-neurotoxins. Non-conventional neurotoxins are not a functionally homogenous group and may target a variety of nicotinic acetylcholine subtypes, display species specific interaction and in some instances their molecular targets remain unknown ${ }^{2,18,31}$. Candoxin produces a novel pattern of neuromuscular blockade at the mammalian neuromuscular junction, not usually associated with curaremimetic snake neurotoxins:(1) the neuromuscular blockade was rapidly and completely reversed by washing or by the addition of an anticholinesterase, and (2) a significant train-of-four fade was observed during the onset of and recovery from neuromuscular blockade. The primary molecular target of candoxin is the postsynaptic muscle $(\alpha \beta \gamma \delta$ nicotinic acetylcholine receptor, while there is evidence that candoxin may also bind to presynaptic autofacilitatory nicotinic acetylcholine receptors to inhibit the positive feedback mechanism of acetylcholine release. Candoxin is also an antagonist of neuronal $\alpha 7$ nicotinic receptors even though it lacks the helix-like conformation of the tip of the middle loop seen in conventional long-chain $\alpha$-neurotoxins and hitherto considered essential for high-affinity binding to $\alpha 7$ receptors.

\section{Clinical and scientific implications}

\section{Application of candoxin in targeted drug delivery for brain tumours}

The blood-brain barrier is a key challenge in the development of drugs for diseases of the central nervous system. Co-delivery of tumour necrosis factor-related apoptosis-inducing ligand (TRAIL) and paclitaxel (PTX) is an attractive treatment strategy for the most aggressive brain tumour, glioblastoma. However, their therapeutic efficacy for intracranial tumours is significantly impaired by blood-brain barrier and bloodtumour barrier ${ }^{44}$. A 16-residue peptide (CDX), derived from candoxin's loop 2 region, was designed and conjugated with TRAIL and paclitaxel-loaded micelles to be utilised as a brain-targeted drug delivery system capable of permeating the blood-brain barrier with great efficacy ${ }^{45,46}$. In vivo bio-distribution and the antiglioblastoma effects observed with this strategy suggest significant potential for the candoxin-derived micelle complex as a novel and effective brain-targeted drug delivery mechanism.

\section{Understanding molecular mechanisms of snake neurotoxin pharmacology}

The significant global morbidity and mortality and consequent economic impact has underpinned the WHO initiative to rank snakebite as a neglected 
tropical disease ${ }^{9,47}$. Sri Lanka has historically been inundated by venomous snakebites and has one of the highest incidence rates of in the world ${ }^{11}$. Nonetheless, universally, the venom composition and pharmacological actions of toxins of many clinically relevant species have remained largely uncharacterized at the molecular level. Greater insight into the mechanisms of $\alpha$-neurotoxin interaction at the mammalian neuromuscular junction will further our understanding of the molecular basis for their clinical envenoming syndromes, with potential for refining current management practices.

\section{Non-conventional neurotoxins as molecular probes and drug leads}

Nicotinic acetylcholine receptors modulate key neuronal functions including neuromuscular transmission, sensory and motor activity, cognition, memory, pain perception and addiction, in addition to playing significant roles in autonomic function and extra-neuronal cholinergic systems in diverse tissues including the lung, blood vessels and epidermis ${ }^{5,6,48-51}$. Consequently, perturbation of cholinergic neurotransmission can result in a variety of neurodegenerative and neurological disorders including myasthenia gravis, Alzheimer's disease, Parkinson's disease, schizophrenia and depression ${ }^{5,51,52}$ and even non-neurological diseases such as lung tumours ${ }^{53}$. The primary challenge to developing nicotinic receptor-targeted therapeutics is the lack of selective ligands as investigative tools or drug leads ${ }^{51}$. Nonconventional neurotoxins $\mathrm{s}^{2,18}$ are a source of highly specific, high-affinity ligands for the multitude of nicotinic receptor subtypes that underpin neurological and neurodegene-rative diseases and have enormous potential to deliver novel applications as scientific probes, diagnostic tools and lead compounds for drug discovery making significant contributions to understanding the human cholinergic circuitry.

\section{Acknowledgements}

I would like to acknowledge the mentorship and contributions of my collaborators Professors M. Kini, J. Sivaraman, M. C. Gwee, P. Gopalakrishnakone (National University of Singapore); J. B. Cohen (Harvard Medical School); J. Tygat (University of Leuven, Belgium); K. Sato (Fukuoka Women's University, Japan); and D. Bertrand (University of Geneva); as well as doctoral students Drs. C. S. Foo, A. Roy and J. Pawlak for their contributions to this body of work. The multiple project and infrastructure research grants from the Biomedical Research Council of Singapore, National University of Singapore Academic Fund, National Neuroscience Institute of Singapore, SingHealth Foundation and Griffith Health Institute are also gratefully acknowledged.

\section{References}

1. Kini RM, Fox JW. Milestones and future prospects in snake venom research. Toxicon 2013; 62: 1-2.

2. Nirthanan S, Gwee MC. Three-finger alpha-neurotoxins and the nicotinic acetylcholine receptor, forty years on. $J$ Pharmacol Sci 2004; 94(1): 1-17.

3. Lewis RJ, Garcia ML. Therapeutic potential of venom peptides. Nat Rev Drug Discov 2003; 2(10): 790-802.

4. Chu NS, Contribution of a snake venom toxin to myasthenia gravis: the discovery of alpha-bungarotoxin in Taiwan. $J$ Hist Neurosci 2005; 14(2): 138-48.

5. Hurst R, Rollema H. Bertrand D, Nicotinic acetylcholine receptors: from basic science to therapeutics. Pharmacol Ther 2013; 137(1): 22-54.

6. Changeux JP. The nicotinic acetylcholine receptor: the founding father of the pentameric ligand-gated ion channel superfamily. J Biol Chem 2012; 287(48): 40207-15.

7. Warrell DA. Redi award lecture: Clinical studies of snakebite in four tropical continents. Toxicon 2012.

8. Warrell DA. Snake bite. Lancet 2010; 375(9708): 77-88.

9. Williams $D$, et al. The Global Snake Bite Initiative: an antidote for snake bite. Lancet 2010; 375(9708): 89-91.

10. Gutierrez JM. Theakston RD, Warrell DA, Confronting the neglected problem of snake bite envenoming: the need for a global partnership. PLoS Med 2006; 3(6): e150.

11. Kasturiratne A, et al. Estimates of disease burden due to land-snake bite in Sri Lankan hospitals. Southeast Asian J Trop Med Public Health 2005; 36(3): 733-40.

12. Del Brutto $\mathrm{OH}$, Del Brutto VJ. Neurological complications of venomous snake bites: a review. Acta Neurol Scand 2012; 125(6): 363-72.

13. Kini RM, Doley R.Structure, function and evolution of threefinger toxins: mini proteins with multiple targets. Toxicon 2010; 56(6): 855-67.

14. Chang CC. Looking back on the discovery of alphabungarotoxin. J Biomed Sci 1999; 6(6): 368-75.

15. Teichert RW, Olivera BM. Natural products and ion channel pharmacology. Future Med Chem 2010; 2(5): 731-44.

16. Puillandre $\mathrm{N}$, et al. Molecular phylogeny, classification and evolution of conopeptides. J Mol Evol 2012; 74(5-6): 297-309.

17. Servent $D$, et al. Only snake curaremimetic toxins with a fifth disulfide bond have high affinity for the neuronal alpha7 nicotinic receptor. J Biol Chem 1997; 272(39): 24279-86.

18. Nirthanan $\mathrm{S}$, et al. Non-conventional toxins from Elapid venoms. Toxicon 2003; 41(4): 397-407.

19. Nirthanan S, et al. Candoxin, a novel toxin from Bungarus candidus, is a reversible antagonist of muscle (alphabetagammadelta ) but a poorly reversible antagonist of neuronal alpha 7 nicotinic acetylcholine receptors. J Biol Chem 2002; 277(20): 17811-20.

20. Nirthanan S, et al. Neuromuscular effects of candoxin, a novel toxin from the venom of the Malayan krait (Bungarus candidus). Br J Pharmacol 2003; 139(4): 832-44. 
21. Nirthanan S, et al. Pharmacological characterization of mikatoxin, an alpha-neurotoxin isolated from the venom of the New-Guinean small-eyed snake Micropechis ikaheka. Toxicon 2002; 40(7): 863-71.

22. Nirthanan $\mathrm{S}$, et al. Biochemical and pharmacological characterization of the venom of the black scorpion Heterometrus spinifer. Biochem Pharmacol 2002; 63(1): 49-55.

23. Nirthanan S, et al. Assignment of voltage-gated potassium channel blocking activity to $\mathrm{k}-\mathrm{KT} \times 1.3$, a non-toxic homologue of k-hefutoxin-1, from Heterometrus spinifer venom. Biochem Pharmacol 2005; 69(4): 669-78.

24. Paaventhan $P$, et al. Crystallization and preliminary X-ray analysis of candoxin, a novel reversible neurotoxin from the Malayan krait Bungarus candidus. Acta Crystallogr D Biol Crystallogr 2003; 59(Pt 3): 584-6.

25. Rani Parvathy $V$, et al. Solution structure of candoxin, a novel three-finger toxin from the venom of Bungarus candidus. ARKIVOC 2006; 15: 1-16.

26. Nirthanan $\mathrm{S}$, et al. Pharmacological characterization of mikatoxin, an $\alpha$-neurotoxin isolated from the venom of the New-Guinean small-eyed snake $<$ i $>$ Micropechis ikaheka $<1$ i>. Toxicon, 2002. 40(7): 863-71.

27. Nirthanan S, et al. Identification of binding sites in the nicotinic acetylcholine receptor for TDBzl-etomidate, a photoreactive positive allosteric effector. J Biol Chem 2008; 283(32): 22051-62.

28. Nirthanan S, et al. Photolabeling the Torpedo nicotinic acetylcholine receptor with 4-azido-2,3,5,6-tetrafluorobenzoylcholine, a partial agonist. Biochemistry 2005; 44(41): 13447-56.

29. Pawlak J, et al. Irditoxin, a novel covalently linked heterodimeric three-finger toxin with high taxon-specific neurotoxicity. FASEB J 2009; 23(2): 534-45.

30. Roy A, et al. Structural and functional characterization of a novel homodimeric three-finger neurotoxin from the venom of Ophiophagus hannah (king cobra). J Biol Chem 2010; 285(11): 8302-15.

31. Torres AM, et al. NMR structure of bucandin, a neurotoxin from the venom of the Malayan krait (Bungarus candidus). Biochem J 2001; 360(Pt 3): 539-48.

32. Servent $D$, et al. Molecular characterization of the specificity of interactions of various neurotoxins on two distinct nicotinic acetylcholine receptors. Eur J Pharmacol 2000; 393(1-3): 197-204.

33. Antil-Delbeke $\mathrm{S}$, et al. Molecular determinants by which a long chain toxin from snake venom interacts with the neuronal alpha 7-nicotinic acetylcholine receptor. $\mathrm{J}$ Biol Chem 2000; 275(38): 29594-601.

34. Rowan EG, et al. Neuromuscular effects of three phospholipases A2 from the venom of the Australian king brown snake Pseudechis australis. Toxicon 1989; 27(5): 551-60.

35. Chang $\mathrm{CC}$. The action of snake venoms on nerve and muscle. In: Snake Venoms 52 ed. Handbook of Experimental Pharmacology, ed. C.-Y. Lee. 1979, Berlin: Springer-Verlag.
36. Marshall IG, Harvey AL. Selective neuromuscular blocking properties of alpha-conotoxins in vivo. Toxicon 1990; 28(2): 231-4.

37. Bowman WC, et al. Feedback control of transmitter release at the neuromuscular junction. Trends Pharmacol Sci 1988; 9(1): 16-20.

38. Fleming TJ, O'HUigin C, Malek TR. Characterization of two novel $L y-6$ genes. Protein sequence and potential structural similarity to alpha-bungarotoxin and other neurotoxins. J Immunol 1993; 150(12): 5379-90.

39. Miwa JM, et al. The prototoxin lynx1 acts on nicotinic acetylcholine receptors to balance neuronal activity and survival in vivo. Neuron 2006; 51(5): 587-600.

40. Tekinay $A B$, et al. A role for $L Y N X 2$ in anxiety-related behavior. Proc Natl Acad Sci U S A 2009; 106(11): 4477-82.

41. Fu XW, Rekow SS, Spindel ER. The ly-6 protein, Iynx1, is an endogenous inhibitor of nicotinic signaling in airway epithelium. Am J Physiol Lung Cell Mol Physiol 2012; 303(8): L661-8.

42. Chernyavsky AI, et al. Novel cholinergic peptides SLURP-1 and-2 regulate epithelialization of cutaneous and oral wounds. Wound Repair Regen 2012; 20(1): 103-13.

43. Ibanez-Tallon I, Nitabach MN. Tethering toxins and peptide ligands for modulation of neuronal function. Curr Opin Neurobiol 2012; 22(1): 72-8.

44. Zhan C, et al. Co-delivery of TRAIL gene enhances the anti-glioblastoma effect of paclitaxel in vitro and in vivo. $J$ Control Release 2012; 160(3): 630-6.

45. Zhan C, et al. Micelle-based brain-targeted drug delivery enabled by a nicotine acetylcholine receptor ligand. Angew Chem Int Ed Engl 2011. 50(24): 5482-5.

46. Zhan C Lu W. The blood-brain/tumor barriers: challenges and chances for malignant gliomas targeted drug delivery. Curr Pharm Biotechnol 2012; 13(12): 2380-7.

47. Gutierrez JM, et al. Snakebite envenoming from a global perspective: Towards an integrated approach. Toxicon, 2010; 56(7): 1223-35.

48. Changeux JP. Allosteric receptors: from electric organ to cognition. Annu Rev Pharmacol Toxicol 2010; 50: 1-38.

49. Corringer $P J$, et al. Structure and pharmacology of pentameric receptor channels: from bacteria to brain. Structure 2012; 20(6): 941-56.

50. Dani JA, Bertrand D. Nicotinic acetylcholine receptors and nicotinic cholinergic mechanisms of the central nervous system. Annu Rev Pharmacol Toxicol 2007; 47: 699-729.

51. D'Hoedt D, Bertrand D. Nicotinic acetylcholine receptors: an overview on drug discovery. Expert Opin Ther Targets, 2009; 13(4): 395-411.

52. Youdim, MB, Buccafusco JJ. Multi-functional drugs for various CNS targets in the treatment of neurodegenerative disorders. Trends Pharmacol Sci 2005. 26(1): 27-35.

53. Paleari L, et al. Inhibition of nonneuronal alpha7-nicotinic receptor for lung cancer treatment. Am J Respir Crit Care Med 2009; 179(12): 1141-50. 